\title{
Glycosylation effects on cancer development
}

\author{
Sen-itiroh Hakomori • Richard D. Cummings
}

(C) Springer Science+Business Media, LLC 2012

Changes in glycosylation associated with oncogenic transformation were first demonstrated with glycosphingolipids over four decades ago. Certain gangliosides, GM3, GD1a and GD1b, which are highly expressed on non-transformed cells, were found to be greatly reduced upon oncogenic transformation $[1,2]$. A subsequent study using a temperaturesensitive mutant of avian sarcoma virus showed close correlation between reduction of GM3 expression and appearance of transformed cell phenotypes. Further study by transfecting GM3-synthase gene into v-Jun transformed mouse and chicken fibroblast cells introduced an important concept that the oncogenic phenotype of cancer cells can be reverted to normal-like phenotype by manipulating the expression level of certain glycosphingolipids [3]. Many studies along similar lines have since been developed with various types of cells.

Numerous studies have demonstrated the close association of various types of $\mathrm{N}$-glycans and O-glycans with cancer development. These findings could potentially be applied to interfere with cancer progression or reversion to normal cell phenotypes. Elevated expression of $\mathrm{N}$ acetylglucosaminyltransferases, such as GnT-III and GnT-V, which are involved in branching modifications

S. Hakomori $(\bowtie)$

Division of Biomembrane Research, Pacific Northwest Research Institute,

720 Broadway,

Seattle, WA 98122, USA

e-mail: hakomori@u.washington.edu

S. Hakomori

Departments of Pathobiology and Global Health, University of

Washington,

Seattle, WA 98185, USA

R. D. Cummings $(\bowtie)$

Department of Biochemistry, Emory University School of

Medicine,

Atlanta, GA 30322, USA

e-mail: rdcummi@emory.edu of N-glycans, has been observed upon cellular transformation [4]. In addition, the truncated O-glycans known as $\mathrm{Tn}$ and its sialylated version Sialyl Tn antigen, are often found to be expressed by many different cancers in immunohistochemical studies, and can arise from altered expression of the enzymes involved in O-glycosylation of proteins [5]. Finally, elevated expression of Lewis-related glycans, such as Sialyl Lewis A on mucin-type glycoproteins of tumor cells has been observed, and is the basis of serum biomarker test for cancer, such as the CA-19-9 antigen [6, 7].

In this specialized volume, entitled "Glycosylation Effects on Cancer Development", we present some of the recent progress and the development of new concepts in this important and growing area.

T. Miyagi and her colleagues describe altered expression of four types of sialidases in cancer cells and focus on Neu3, which has enhanced expression in various cancer cells. Neu3 is known to specifically hydrolyze gangliosides including GM3, but not sialyl groups of other glycans, and is indicated to stimulate cell growth through interacting with growth factor receptors. In addition, the authors discuss their result that the knockdown of Neu3 expression by siRNA causes apoptosis in cancer cells, and suggest Neu3 could be a useful target for inhibition of cancer progression.

K. Furukawa's group summarizes their studies on GD3 and GD2, which are highly expressed in melanomas and small cell lung cancer cells. They found that the gangliosides enhance cell motility and invasiveness by stimulating focal adhesion kinase and Lyn kinase, which phosphorylate paxillin and p130Cas. Further studies using the reconstructed membrane system indicated GD3 and GD2 function in glycosphingolipid- enriched microdomains/rafts. This line of study may eventually lead to a novel approach to block cancer metastasis.

S. Sonnino and his colleagues report, for the first time, the effect of ionizing radiations on plasma membrane- 
associated glycosidases including Neu3. Their studies, focused on human breast cancer cells, showed that the irradiation also causes an increase of apoptosis and plasma membrane ceramide. Further studies in this line may provide a new direction in this area of cancer research.

J. Gu's group summarizes recent advances in the potential role of aberrant $\mathrm{N}$-glycosylation in integrin-mediated cell adhesion and migration, focusing on bisecting GnT-III and GnT-V necessary for multi-antennary structures. They discuss possibly competing functions of GnT-III and GnT-V on cancer progression. Additional studies will further clarify this point.

P. Stanley, R. Cummings and colleagues discuss a role of GnT-III in cancer development from the view that the newly-introduced bisecting GlcNAc in complex or hybrid $\mathrm{N}$-glycans confers unique recognition properties for lectins including galectins. The altered recognition with some lectins may be due to conformational changes induced by the bisecting GalNAc. They summarize results indicating that the bisecting GlcNAc modulates cell growth through the interaction between galectins and $\mathrm{N}$-glycans on growth factor receptors.

C. Dimitroff's group discuss the possible mechanisms for how cancer cells escape anti-tumor immune response of cytotoxic $\mathrm{CD} 8(+) \mathrm{T}$ cells. They describe results showing that tumor-derived galectins and concomitant presence of their ligands on $\mathrm{CD} 8(+) \mathrm{T}$ cells function as negative regulators of anti-tumor immunity, perhaps through apoptosis of the immune cells. They propose that galectins released from cancer cells bind to CD7 or CD45 on $\mathrm{CD} 8(+) \mathrm{T}$ cells, inducing apoptosis of the immune cells.

S. Hakomori and his colleague describe a novel molecular interaction between carbohydrate epitopes, termed carbohydrate-carbohydrate interaction (CCI). In addition to previous studies focusing on the involvement of CCIs in cell-to-cell interaction, CCIs between molecules expressed within the same membrane microdomain were shown to provide the molecular mechanism for effects of gangliosides on growth factor receptor-associated tyrosine kinases.

\section{References}

1. Hakomori, S., Murakami, W.T.: Proc Natl Acad Sci USA 59(1), 254-261 (1968)

2. Mora, P.T., Brady, R.O., Bradley, R.M., McFarland, V.W.: Proc Natl Acad Sci USA 63(4), 1290-1296 (1969)

3. Miura, Y., Kainuma, M., Jiang, H., Velasco, H., Vogt, P.K., Hakomori, S.: Proc Natl Acad Sci USA 101(46), 16204-16209 (2004)

4. Lau, K.S., Dennis, J.W.: Glycobiology 18(10), 750-760 (2008)

5. Ju, T., Otto, V.I., Cummings, R.D.: Angew Chem Int Ed Engl 50(8), 1770-1791 (2011)

6. Kannagi, S., Sakuma, K., Miyazaki, K., Lim, K.T., Yusa, A., Yin, J., Izawa, M.: Cancer Sci 101(3), 586-593 (2009)

7. Goonetilleke, K.S., Siriwardena, A.K.: Eur J Surg Oncol 33(3), 266-270 (2007) 